\title{
Graphene oxide as nano reinforcing material in cement-based composites:
}

\section{A review}

\author{
S. Chiranjiakumari Devi ${ }^{1}$ and Rizwan A. Khan ${ }^{1 *}$ \\ ${ }^{1}$ Deptt. of civil Engg., Dr. B. R. Ambedkar NIT Jalandhar, Punjab 144011, India. \\ *Email:khanar@nitj.ac.in/rizwankhan1@gmail.com
}

Nanotechnology has become one of the most active research areas in construction materials. Nano composites such as CNTs, Graphene Oxide (GO)derived graphene-based materials are being used in various disciplines as they enhances both mechanical, electrical properties and also transport properties determining the long term durability in concrete structure. But nanomaterial generally agglomerates due to the strong Van der Waal's forces at nano scale and reduced workability which may be due to adsorption of free water onto the surface of nanomaterial in concrete which may be overcome by a perfect combination of sonication for mechanical separation and compatible admixtures of the respective nanocomposites [1]. It has been observed that graphene oxide which is a monolayer of graphene with oxygen containing functional groups [5] has the ability to absorb water molecule producing a water reservoir and water transport channel for further hydration of cement, accelerates the hydration rate due to its catalytic behavior when added to cement as reinforcing material [2]. Effects of GO addition into cement matrix can effectively improve the transport properties in cement which eventually improve its durability (its transport properties reduce the movement of aggressive chemicals due to formation of strong barrier enhancing its resistance to aggressive elements). With surface area larger than that of cement makes GO a filler material in nano scale refining the pore structure which enhances the compressive strength by $13-$ $33 \%$, flexural strength by $41-58 \%$, increasing the viscosity sharply and reducing the setting time of cement mortar with increment of GO and with ages. Figure 1 illustrates the SEM images of hydrated cement paste at 28 days showing flowerlike crystals. The amount of crystals increases with increase in concentration of GO in cement matrix resulting in the formation of reinforced structure by reducing harmful pores $[3,4]$. Comparing the limits of nanomaterial composite i.e. CNTs, Graphene - derived cement based (gets agglomerated easily) and Graphene oxide (GO) of which GO cement composite shows a better result being surface-functionalized in nature having very less dispersion problem and the reduced setting time which is not more than 30 minutes making the cement usable as it does not exceed the range of national standard.
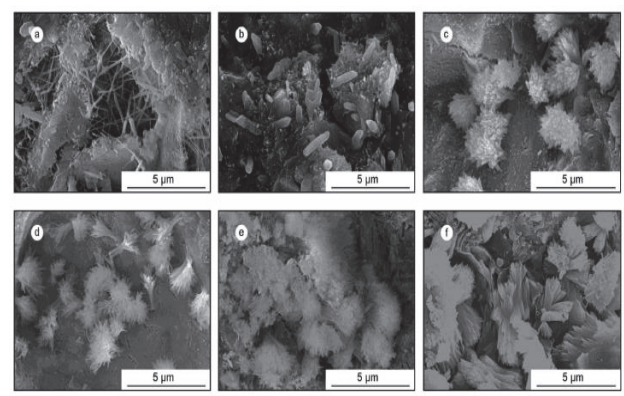

Figure 1: SEM images of hardened cement paste at 28 days in addition of 0 to $0.05 \%$ of GO (by wt. of cement) with an increment of $0.01 \%$ in (a), (b), (c), (d), (e) and (f) respectively [2].

This paper gives an overview about the limits and influence of the nanomaterial $(\mathrm{GO})$ as reinforcing material in cement based composites due to its dispersibility property, performance in microstructure level to enhancement of mechanical properties by controlling the cracks at nano scale level and improving the microstructure of cement matrix, transport properties and durability governing the service life of the concrete structures.

\section{References}

1. A. Mohammed, J.G. Sanjayan, W.H. Duan, A. Nazari, Construction and Building Materials 84 (2015) 341-347.

2. Changqing Lin, WeiWei, YunHangHu, Journal of Physics and Chemistry of Solids 89 (2016) 128-133.

3. Qin Wang, Jian Wang, Chun-xiang Lu, Bo-wei Liu, Kun Zhang, Chong-zhi Li, New Carbon Materials, 2015, 30(4): 349-356.

4. Zhu Pan, Li He, Ling Qiu, Asghar Habibnejab Koravem, Gang Li, Jun Wu Zhu, Frank Collins, Dan Li, Wen Hui Duan, Ming Chien Wang, Cement \& Concrete Composites 58 (2015) 140-147.

5. Snigdha Sharma and N. C. Kothiyal, RSC Adv., 2015, 5, 52642-52657. 